\title{
Análisis de Perfiles Cognitivos en Población Clínica Infantil con Trastornos del Neurodesarrollo
}

\author{
Analysis of Cognitive Profiles in Infant Clinical Population with \\ Neurodevelopmental Disorders
}

\author{
Julia Vacas ${ }^{1}$, Adoración Antolî́2, Araceli Sánchez-Raya ${ }^{3}$ y Fátima Cuadrado ${ }^{4}$
}

\begin{abstract}
Resumen
Dada su común afectación en el lenguaje, este estudio pretende explorar el perfil cognitivo asociado al Trastorno Específico del Aprendizaje, Trastorno Específico del Lenguaje, Síndrome de Asperger, Trastorno por Déficit de Atención e Hiperactividad subtipos Inatento y Combinado formulando criterios de exclusión que orienten el diagnóstico. Se contó con 156 casos clínicos de entre 6 y 15 años $(M=9.5, D T=2.18)$ evaluados con WISC-IV, considerando como variables dependientes los índices comprensión verbal, razonamiento perceptivo, memoria de trabajo, velocidad de procesamiento y CI total. El análisis de datos se centró en detectar diferencias intergrupo e intragrupo. Los resultados muestran que el Trastorno Específico del Lenguaje presenta un perfil distintivo respecto a los demás. También se observa que generalmente memoria de trabajo es el índice más afectado, mientras que razonamiento perceptivo se mantiene intacto. Estas conclusiones repercuten en la práctica clínica permitiendo concretar distinciones entre trastornos e individualizar intervenciones.
\end{abstract}

Palabras clave: perfil cognitivo, trastornos del neurodesarrollo, WISC-IV, análisis de discrepancias, diagnóstico

\begin{abstract}
Considering their common affection in certain language dimensions, this study attempts to explore cognitive profiles associated with Specific Learning Disorders, Specific Language Impairment, Asperger Syndrome and Attention-Deficit/Hyperactivity Disorder in two dimensions (Predominantly Inattentive and Combined). The sample consisted of a clinical population of 156 children between 6 and 15 years old $(M=9.5, S D=2.18)$ who were applied WISC-IV. The four indexes from this scale (verbal comprehension, perceptual reasoning, working memory and processing speed) and the full-scale intelligence quotient were considered as dependent variables. Data analysis was focused on the assessment of intergroup and intragroup differences. Results prove a highly distinctive profile for Specific Language Impairment compared to the other disorders and a general pattern of strengths and weaknesses among them. Conclusions from this study contribute to the improvement of clinical practice as our data highlight significant differences between neurodevelopmental disorders which may drive to more individualised interventions.
\end{abstract}

Keywords: cognitive profile, neurodevelopmental disorders, WISC-IV, pairwise discrepancies analysis, diagnostic

\footnotetext{
${ }^{1}$ Departamento de Psicología, Universidad de Córdoba. Instituto Maimónides de Investigación Biomédica de Córdoba (IMIBIC). Hospital Universitario Reina Sofía de Córdoba, España. Correspondencia: Facultad de Ciencias de la Educación, Universidad de Córdoba; C/ San Alberto Magno, s/n, 14071, Córdoba, España. Tel.: 658436077. Correo: 172varuj@uco.es

${ }^{2}$ Departamento de Psicología, Universidad de Córdoba. Instituto Maimónides de Investigación Biomédica de Córdoba (IMIBIC). Hospital Universitario Reina Sofía de Córdoba, España. Centro de Atención Infantil Temprana de la Universidad de Córdoba (CAITUCO), España.

${ }^{3}$ Departamento de Psicología, Universidad de Córdoba. Instituto Maimónides de Investigación Biomédica de Córdoba (IMIBIC). Hospital Universitario Reina Sofía de Córdoba, España. Centro de Atención Infantil Temprana de la Universidad de Córdoba (CAITUCO), España.

${ }^{4}$ Departamento de Psicología, Universidad de Córdoba. Instituto Maimónides de Investigación Biomédica de Córdoba (IMIBIC). Hospital Universitario Reina Sofía de Córdoba, España.
}

Revista Iberoamericana de Diagnóstico y Evaluación - e Avaliação Psicológica. RIDEP · №54 · Vol.1 · 35-46 2020 ISSN: 1135-3848 print /2183-6051online 
Introducción

\section{Trastornos del Neurodesarrollo}

El Manual Diagnóstico y Estadístico de los Trastornos Mentales (5th ed.; DSM-V; American Psychiatric Association, 2013) define los Trastornos del Neurodesarrollo (TND) como un conjunto de afecciones que aparecen precozmente en el curso del desarrollo infantil interfiriendo en el mismo y causando déficits en la manera en que el niño se desenvuelve en los ámbitos personal, social, académico u ocupacional. El propio manual insiste en que una de las características que mejor define los TND es la variabilidad tanto en las áreas afectadas como en el grado de afectación de las mismas, dando como resultado un amplio abanico de combinaciones y posibilidades que el profesional clínico debe manejar correctamente para ofrecer un diagnóstico acertado. Así, se puede afirmar que generalmente los niños no presentan un perfil clínico claro, es decir, no muestran todas las características asociadas a un trastorno concreto, sino que pueden manifestar rasgos de distintas patologías. Todo ello incrementa la dificultad de realizar un diagnóstico adecuado, por ello el propio DSM-5 recomienda revisarlo periódicamente para conocer su evolución por si pudiera haber evolucionado a un perfil clínico distinto. Dadas las dificultades mencionadas, resulta imprescindible tanto conocer las características propias de los diferentes trastornos como establecer comparaciones entre aquellos que presenten rasgos muy similares. Para evitar confusiones y diagnósticos erróneos, desde la investigación se deben proporcionar criterios diferenciales que guíen el diagnóstico y faciliten la toma de decisiones.

\section{WISC-IV como Herramienta para la Evaluación de los TND}

Este trabajo no está orientado exclusivamente al proceso diagnóstico, sino que también pretende generar reflexión sobre el papel que juegan los datos obtenidos en las pruebas psicométricas para la fundamentación de las intervenciones terapéuticas. Es bien sabido que en la intervención con personas con TND se deben tener en consideración tanto las fortalezas como las debilidades cognitivas del sujeto de manera que se favorezca el aprovechamiento de las primeras para reforzar y complementar las segundas. En este sentido, las nuevas técnicas de neuroimagen y los modelos computacionales han demostrado que las áreas del cerebro están en permanente conexión y evolución, de manera que los procesos de desarrollo y maduración cognitiva poseen un carácter dinámico e interactivo en el cual las diferentes áreas compiten y cooperan entre ellas (Wass, 2015).

Otro elemento fundamental de este estudio ha sido el empleo de la Escala de Inteligencia para Niños de Weschler versión 4 (WISC-IV; Weschler, 2003a). Esta batería cognitiva destaca como la principal herramienta clínica empleada para la detección y el diagnóstico de trastornos en la infancia según la opinión y la experiencia de los profesionales tanto a nivel europeo (Evers et al., 2012) como concretamente en España (Muñiz \& Fernández-Hermida, 2010). Igualmente, es la escala más utilizada como instrumento de evaluación neuropsicológica en Estados Unidos y Canadá (Rabin, Paolillo, \& Barr, 2016). Según estos autores, el WISC-IV ocupa la quinta posición en la categoría general de "instrumentos de evaluación usados con mayor frecuencia", concretamente destaca en la evaluación específica de habilidades como atención, concentración, memoria de trabajo, inteligencia general, logro, habilidades visoespaciales, e incluso el estado mental y la cognición global. De este modo, se comprueba que a pesar de la actual controversia sobre la adecuación del WISC-IV como herramienta de evaluación y diagnóstico, su utilización en la práctica clínica está ampliamente extendida.

\section{Estudios Previos sobre Perfiles Cognitivos en TND}

En el presente estudio se han seleccionado un conjunto de trastornos que tienen en común alteraciones en determinadas áreas del lenguaje; si bien las habilidades lingüísticas alteradas son distintas en cada trastorno, en muchos casos la afectación puede manifestarse de formas similares, repercutiendo considerablemente en la concreción de su diagnóstico. Los trastornos seleccionados han sido: Trastorno Específico del Aprendizaje, concretamente Dificultades en el Aprendizaje de la Lectura o dislexia (DAL), Trastorno Específico del Lenguaje (TEL), 
Síndrome de Asperger (SA), Trastorno por Déficit de Atención e Hiperactividad predominantemente Inatento (TDAH-I) y su variante de tipo Combinado (TDAH-C). Otro motivo que justifica la selección de trastornos es la cantidad de estudios que resaltan una alta comorbilidad entre algunos de ellos, como es el caso de TEL-TDAH (Gómez, Vance, \& Watson, 2016; Miranda-Casas, Soriano-Ferrer, \& Baixauli-Fortea, 2011) y TELDAL (Schuchardt, Bockmann, Bornemann, \& Maehler, 2013).

Hasta la fecha, múltiples trabajos han tratado de esclarecer los perfiles cognitivos asociados a las patologías mencionadas. Sin embargo, ninguno de ellos ha abarcado tal variedad de trastornos. No obstante, las conclusiones derivadas de estos estudios previos suponen el punto de partida de esta investigación. Así, se puede definir a la población con DAL por sus déficits en memoria de trabajo y velocidad de procesamiento (Berninger \& O’Donell, 2005; Cornoldi, Giofre, Orsini, \& Pezzuti, 2014; Giofrè \& Cornoldi, 2015). Por otra parte, también se ha hallado una alta comorbilidad entre DAL y TEL matizando que ambas patologías presentan afectación en memoria de trabajo, aunque dentro de esta función cognitiva, son distintas las áreas que aparecen afectadas en uno y otro trastorno (Schuchardt et al., 2013). Por ello, el estudio en profundidad de este constructo podría ayudar a discernir entre una patología y otra.

Por otro lado, las dificultades en la memoria de trabajo son consideradas como un marcador fenotípico fiable para predecir la presencia de TEL (Conti-Ramsden, 2003; Lum, Ullman, \& Conti-Ramsden, 2015; Schuchardt et al., 2013; Spanoudis \& Natsopoulos, 2011). Sin embargo, algunos estudios ponen en duda esta afirmación al señalar que existe un porcentaje considerable de niños TEL (entre un 20 y un 25\%) que no presentan afectación en este índice (Alloway, Rajendran, \& Archibald, 2009) o encontrar la memoria de trabajo intacta en una muestra de prescolares con TEL que presentaban alteración en la velocidad de procesamiento (Reichenbach, Bastian, Rohrbach, Gross, \& Sarrar, 2016). Por otro lado, respecto a comprensión verbal, numerosos trabajos evidencian que existen importantes déficits en este grupo en cuanto a habilidades lingüísticas fundamentales como la conciencia metalingüística y la comprensión de verbos mentales (Spanoudis \& Natsopoulos, 2011) o la adquisición de estructuras gramaticales, morfológicas y sintácticas complejas (CastroRebolledo et al., 2004; van Weerdenburg, Verhoeven, \& van Balkom, 2006). Todo ello permite hipotetizar que el constructo comprensión verbal estará notablemente afectado, aunque ningún estudio hasta el momento ha obtenido resultados claramente significativos empleando la escala WISC-IV.

Por su parte, el perfil cognitivo de la población con SA destaca por ser ampliamente heterogéneo, con picos que sobresalen la media y habilidades cognitivas significativamente por debajo de la norma (González-Gadea et al., 2014). En general, la investigación coincide en resaltar los déficits de estos sujetos en memoria de trabajo y velocidad de procesamiento (Oliveras-Rentas, Kenworthy, Roberson, Martin, \& Wallace, 2012; Planche \& Lemonnier, 2012), así como un patrón de comprensión verbal notablemente superior al razonamiento perceptivo que permite diferenciar a esta población de otros tipos de trastornos del espectro autista (Nedelcu \& Buceta, 2012).

Finalmente, la población TDAH muestra también una clara afectación en los índices memoria de trabajo y velocidad de procesamiento; sin embargo, no existe consenso acerca de su alcance. Algunos estudios defienden que dicha afectación está presente en ambos índices y subtipos: inatento y combinado (Bustillo \& Servera, 2015) e incluso encuentran un patrón diferenciado entre ambos ya que el TDAH-I presentaría mayores déficits en memoria de trabajo que en velocidad de procesamiento, mientras que en TDAH-C la tendencia es a la inversa (Fenollar-Cortés, Navarro-Soria, González-Gómez, \& García-Sevilla, 2015); otros solo encuentran dificultades en memoria de trabajo sin distinción entre un subtipo y otro (García et al., 2013; Miranda-Casas et al., 2011); y un tercer grupo sugiere que solo el TDAH-I muestra bajo rendimiento en los índices mencionados (Capdevila-Brophy, ArtigasPallarés, \& Obiols-Llandrich, 2006).

A la luz de la información expuesta, se comprueba que los estudios citados muestran resultados y conclusiones muy diferentes en cuanto al perfil cognitivo de estas poblaciones; de 
Tabla 1. Características de la Muestra

\begin{tabular}{|c|c|c|c|c|c|c|c|c|c|c|c|c|c|}
\hline \multirow{2}{*}{\multicolumn{2}{|c|}{ Variable }} & \multicolumn{2}{|c|}{$\begin{array}{c}\text { DAL } \\
(n=25)\end{array}$} & \multicolumn{2}{|c|}{$\begin{array}{c}\text { TEL } \\
(n=25)\end{array}$} & \multicolumn{2}{|c|}{$\begin{array}{c}\text { SA } \\
(n=35)\end{array}$} & \multicolumn{2}{|c|}{$\begin{array}{c}\text { TDAH-I } \\
(n=35)\end{array}$} & \multicolumn{2}{|c|}{$\begin{array}{c}\text { TDAH-C } \\
(n=36)\end{array}$} & \multicolumn{2}{|c|}{$\begin{array}{c}\text { Total } \\
(N=156)\end{array}$} \\
\hline & & $f$ & $\%$ & $f$ & $\%$ & $f$ & $\%$ & $f$ & $\%$ & $f$ & $\%$ & $f$ & $\%$ \\
\hline \multirow[t]{3}{*}{ Sexo } & Niño & 19 & $12.2 \%$ & 22 & $14.1 \%$ & 33 & $21.2 \%$ & 25 & $16 \%$ & 27 & $17.3 \%$ & 126 & $80.8 \%$ \\
\hline & Niña & 6 & $3.8 \%$ & 3 & $1.9 \%$ & 2 & $1.3 \%$ & 10 & $6.4 \%$ & 9 & $5.8 \%$ & 30 & $19.2 \%$ \\
\hline & Total & 25 & $16 \%$ & 25 & $16 \%$ & 35 & $22.4 \%$ & 35 & $22.4 \%$ & 36 & $23.1 \%$ & 156 & $100 \%$ \\
\hline \multirow[t]{6}{*}{ Edad } & 6-7 & 7 & $4.5 \%$ & 10 & $6.4 \%$ & 5 & $3.2 \%$ & 5 & $3.2 \%$ & 6 & $3.8 \%$ & 33 & $21.2 \%$ \\
\hline & 8-9 & 10 & $6.4 \%$ & 3 & $1.9 \%$ & 18 & $11.5 \%$ & 12 & $7.7 \%$ & 11 & $7.7 \%$ & 55 & $35.2 \%$ \\
\hline & $10-11$ & 6 & $3.8 \%$ & 9 & $5.8 \%$ & 4 & $2.6 \%$ & 8 & $5.1 \%$ & 9 & $5.8 \%$ & 36 & $23.1 \%$ \\
\hline & $12-13$ & 2 & $1.3 \%$ & 3 & $1.9 \%$ & 5 & $3.2 \%$ & 8 & $5.1 \%$ & 6 & $3.9 \%$ & 24 & $15.4 \%$ \\
\hline & $14-15$ & 0 & $0 \%$ & 0 & $0 \%$ & 3 & $1.9 \%$ & 2 & $1.3 \%$ & 3 & $1.9 \%$ & 8 & $5.1 \%$ \\
\hline & Total & 25 & $16 \%$ & 25 & $16 \%$ & 35 & $22.4 \%$ & 35 & $22.4 \%$ & 36 & $23.1 \%$ & 156 & $100 \%$ \\
\hline
\end{tabular}

ahí que se mantenga la necesidad de seguir investigando en esta temática con objeto de obtener datos más concluyentes. En este sentido, el objetivo general de este trabajo es ampliar y mejorar las posibilidades del WISC-IV como herramienta complementaria para orientar el diagnóstico y que sirva como punto de partida para al diseño de la intervención específica con niños con TND. Para lograrlo, se ha evaluado y comparado los perfiles cognitivos concretos de los TND seleccionados, estableciendo diferencias intergrupo que faciliten el diagnóstico diferencial. Paralelamente, con el objetivo de establecer diferencias intragrupo, se han analizado las discrepancias entre pares de índices. En otras palabras, los dos objetivos específicos del estudio se centran en el análisis intergrupo e intragrupo de los perfiles cognitivos de las poblaciones seleccionadas como medio para expandir la aplicabilidad del WISC-IV durante el proceso diagnóstico. La hipótesis de partida apunta a que, a nivel intergrupo, los índices memoria de trabajo y velocidad de procesamiento serán los más vulnerables en todos los trastornos evaluados, en el caso de la población TEL podría encontrarse también afectación en comprensión verbal (H1). Igualmente, se espera encontrar diferencias entre trastornos según el grado de afectación de tales índices, siendo dicha afectación mayor en los sujetos TEL, SA y TDAH y menor en DAL (H2). A nivel intragrupo, la hipótesis principal sugiere que SA mostrará un perfil más heterogéneo, diferenciándose así del resto de trastornos (H3).

\section{Método}

\section{Participantes}

A partir del software GPower y estableciendo un tamaño del efecto de .06 , un error $\alpha$ de .05 y un error $\beta$ de .95 se determinó que la muestra total debería incluir un mínimo de 130 participantes para poder realizar los análisis adecuados, de manera que se recogieron datos de 156 casos evaluados en la Unidad de Salud Mental Infantojuvenil (USMIJ) del Hospital Reina Sofía de Córdoba (Tabla 1). La principal variable definitoria del grupo muestral fue el diagnóstico, seguido por la edad. Como se puede observar en la Tabla 1, los sujetos examinados oscilan entre 6 y 15 años $(M=9.5, D T=2.18, E T=.18)$. Por otro lado, respecto al sexo, la muestra concuerda con las conclusiones derivadas de la investigación epidemiológica en este tipo de trastornos que resalta una mayor afectación entre la población masculina, en torno a 2.8:8 a favor de las mujeres (Castro-Rebodello, Giraldo-Prieto, HincapiéHenao, Lopera, \& Pineda, 2004).

\section{Instrumento}

El WISC-IV (Wechsler, 2003a) es el resultado de la revisión y actualización de una larga tradición de pruebas de inteligencia diseñadas por David Wechsler que han tenido como objetivo superar el mero uso de las escalas como tests psicométricos (Flanagan \& Kaufman, 2006). Recoge información sobre cuatro aspectos básicos de la inteligencia: comprensión verbal (CV), razonamiento perceptivo (RP), memoria de trabajo (MT) y velocidad de procesamiento (VP) a 
partir de quince tests (diez principales y cinco optativos). Está dirigido a población con edades comprendidas entre 6 y 16 años.

Existen numerosos estudios que han puesto a prueba las propiedades psicométricas de esta escala, entre ellas su tipificación, fiabilidad, saturación en $g$ y validez estructural (Flanagan \& Kaufman, 2006). Del mismo modo, esta batería de tests fue sometida a un importante procedimiento de tipificación que contó con 2200 sujetos en la versión estadounidense y 1590 en la española (Corral, Arribas, Santamaría, Sueiro, \& Pereña, 2005). En ambos casos se ofrecen resultados basados en grupos especiales que resultan de gran interés para obtener un marco de referencia con el que comparar puntuaciones.

La información obtenida en esta escala es muy variada, ya que ofrece datos que abarcan desde el CI total hasta medidas resultantes de la combinación de índices como el ICG (Índice de Capacidad General) derivado de la suma de las puntuaciones escalares de las subpruebas de los índices CV y RP y su correspondencia con los baremos del manual de interpretación de la escala, o el ICC (Índice de Competencia Cognitiva) obtenido a partir de la suma de las puntuaciones escalares de las subpruebas de los índices MT y VP y su correspondencia con los baremos del manual de interpretación de la escala. Estos índices han sido ampliamente utilizados en la investigación de perfiles clínicos, por lo que en el presente estudio se ha ampliado este análisis de discrepancias abarcando la comparación de pares de índices (véase en procedimiento y análisis estadístico).

\section{Procedimiento y Análisis Estadístico}

La selección de casos se realizó en función del diagnóstico entre la población clínica infantil atendida y evaluada por los profesionales especialistas acreditados en psicología clínica sanitaria de la USMIJ del Hospital Reina Sofía de Córdoba siguiendo el protocolo establecido en la unidad para tal efecto. Durante el proceso de recogida de datos, se garantizó que toda la información fuera tratada de forma anónima y confidencial de acuerdo con el Real Decreto-ley $5 / 2018$, de 27 de julio, de medidas urgentes para la adaptación del Derecho español a la normativa de la Unión Europea en materia de protección de datos y siguiéndose de forma rigurosa los principios éticos promulgados en la declaración de Helsinki por la Asociación Médica Mundial. Igualmente, se obtuvo la aprobación y el consentimiento del Comité de Ética de la Investigación de Córdoba para el desarrollo del proyecto.

Tras recoger las puntuaciones de los sujetos seleccionados en las diferentes pruebas e índices de la batería cognitiva, estas fueron tratadas con el Statistical Package for the Social Sciences, 21 (SPSS, 21) con el que se llevó a cabo un análisis intergrupo. Así, se realizó un análisis multivariante de la varianza (MANOVA) introduciendo como factor fijo el diagnóstico y como variables dependientes los cuatro índices del WISC-IV (CV, RP, MT y VP) y el CIT para obtener un perfil cognitivo concreto y bien diferenciado de cada patología. Igualmente, para aislar cualquier posible efecto del sexo sobre los resultados, éste fue incorporado al análisis como covariable.

Por otro lado, también se llevó a cabo un análisis intragrupo de estos perfiles por medio de la mencionada comparación de discrepancias entre pares de índices. Dichas discrepancias entre los índices marcan la heterogeneidad del perfil y son una señal que indica la posibilidad de que exista un trastorno o el grado de afectación del mismo. Así, se pusieron en relación los distintos índices y se observaron las diferencias entre ellos a fin de comprobar si existe un fuerte contraste entre el rendimiento del sujeto en una habilidad cognitiva específica sobre las demás. Las parejas de índices contrastadas fueron: CV-RP, CV-MT, CV-VP, RP-MT, RP-VP, MT-VP.

Las comparaciones entre las puntuaciones individuales de un sujeto en cualquier prueba psicométrica y el contraste del perfil de dicho sujeto con las puntuaciones normativas son procedimientos fundamentales en la práctica neuropsicológica ya que ayudan a detectar y cuantificar la dimensión del déficit (Crawford, Garthwaite, \& Gault, 2007). Existen diversos protocolos para el tratamiento de las comparaciones entre índices y el establecimiento de diferencias anormales. En este caso, se ha escogido el enfoque empírico del manual ya que la muestra de estandarización del WISC-IV es muy amplia y el propio proceso de estandarización ofrece una 


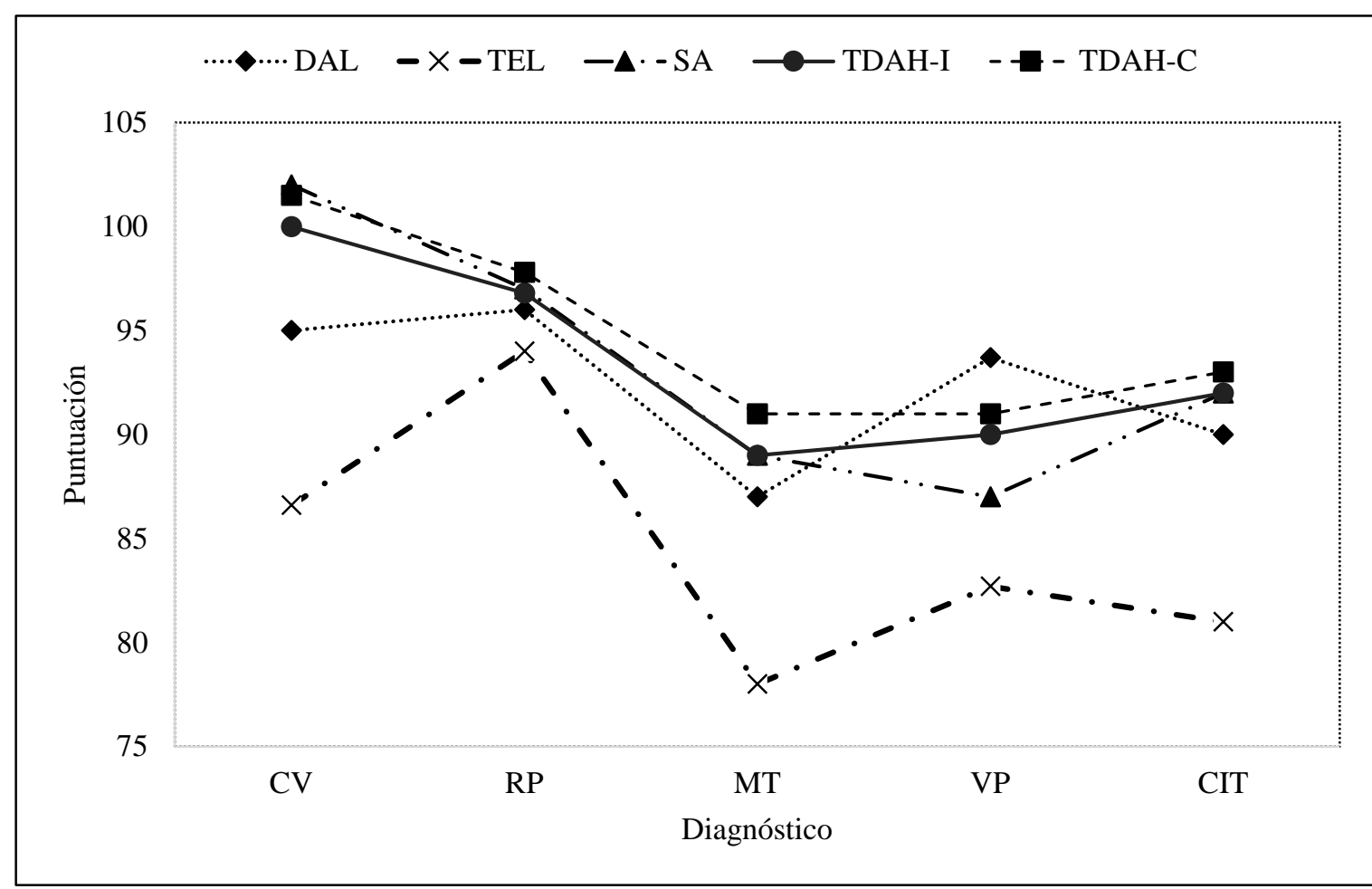

Figura 1. Diferencias Intergrupo atendiendo al Perfil Cognitivo en puntuaciones en las subescalas del WISC-IV

distribución normal multivariada (Crawford, Anderson, Rankin, \& MacDonald, 2010).

El procedimiento basado en el enfoque empírico consiste en hallar la diferencia entre las puntuaciones compuestas de las parejas de índices y comparar el valor resultante con los valores críticos aportados en los apéndices del manual (Tabla B.2). En este proceso, se tiene en cuenta la edad de los sujetos, el valor positivo o negativo de la diferencia hallada (éste marca la dirección a favor de un índice u otro) y el nivel de confianza (85\% o 95\%) que se emplea para la detección de discrepancias significativas. Todo ello con objeto de dar un paso más en la concreción de los perfiles cognitivos afinando las diferencias intragrupo.

\section{Resultados}

\section{Análisis Intergrupo}

La Figura 1 permite apreciar de una manera gráfica los resultados obtenidos en cada perfil. Así, se observa que los sujetos TEL muestran un perfil cognitivo significativamente más bajo que el resto de los trastornos estudiados cuyas puntuaciones medias son bastante similares en índices como RP, MT y CIT. Los índices CV y VP muestran más variabilidad entre trastornos, aunque manteniéndose dentro de los límites que definen a la población normativa $(M=100, D T= \pm 15)$; $\sin$ embargo, en el caso de TEL, esta población se encuentra por debajo de estos límites en todos los índices medidos excepto en CV y RP.

Analizando más detenidamente la figura 1, se puede ver que: en primer lugar, $\mathrm{CV}$ es el índice más destacado (por encima de 100) en SA y en ambos subtipos de TDAH. Por otra parte, RP es el punto fuerte en sujetos TEL y DAL. Por el contrario, MT es el índice que muestra puntuaciones más bajas en todos los trastornos estudiados, exceptuando SA; en el caso de los sujetos TEL, este índice se configura claramente como un punto débil, ya que se aleja de la media en más de 20 puntos. Por otro lado, los resultados de la VP muestran que este es el índice con menor rendimiento entre la población SA. Finalmente, el CIT de los sujetos DAL, SA, TDAH-I y TDAH-C es bastante similar y se mueve dentro de los parámetros considerados normales, mientras que los sujetos TEL presentan un CIT por debajo de la norma. De esta forma, la $\mathrm{H} 1$ se ve parcialmente confirmada ya que MT y VP son los índices que presentan puntuaciones más bajas en general, aunque solo en el caso de TEL las puntuaciones son 
Tabla 2. Análisis de Diferencias Intergrupo. MANOVA para Trastornos e Índices

\begin{tabular}{|c|c|c|c|c|c|c|c|c|}
\hline \multirow{3}{*}{ Variable } & \multicolumn{5}{|c|}{ Diagnóstico } & \multirow{3}{*}{$\begin{array}{c}F \\
(4,146)\end{array}$} & \multirow[b]{3}{*}{$p$} & \multirow{3}{*}{$\eta^{2}$} \\
\hline & DAL & TEL & SA & TDAH-I & TDAH-C & & & \\
\hline & $\begin{array}{c}\mathrm{M} \\
(\mathrm{DT}, \mathrm{ET})\end{array}$ & $\begin{array}{c}\mathrm{M} \\
(\mathrm{DT}, \mathrm{ET})\end{array}$ & $\begin{array}{c}\mathrm{M} \\
(\mathrm{DT}, \mathrm{ET})\end{array}$ & $\begin{array}{c}\mathrm{M} \\
(\mathrm{DT}, \mathrm{ET})\end{array}$ & $\begin{array}{c}\mathrm{M} \\
(\mathrm{DT}, \mathrm{ET})\end{array}$ & & & \\
\hline $\mathrm{CV}$ & $\begin{array}{c}95.20 \\
(13.33,2.66)\end{array}$ & $\begin{array}{c}86.60 \\
(11.59,2.66)\end{array}$ & $\begin{array}{c}102.57 \\
(10.80,2.27)\end{array}$ & $\begin{array}{c}100.29 \\
(16.07,2.26)\end{array}$ & $\begin{array}{c}101.44 \\
(13.55,2.22)\end{array}$ & 6.92 & .000 & .16 \\
\hline RP & $\begin{array}{c}96.44 \\
(15.40,2.61)\end{array}$ & $\begin{array}{c}94.08 \\
(15.03,2.61)\end{array}$ & $\begin{array}{c}97.14 \\
(12.22,2.23)\end{array}$ & $\begin{array}{c}96.89 \\
(10.50,2.21)\end{array}$ & $\begin{array}{c}97.86 \\
(12.55,2.17)\end{array}$ & .32 & .867 & .01 \\
\hline MT & $\begin{array}{c}87.32 \\
(15.42,2.57)\end{array}$ & $\begin{array}{c}78.08 \\
(13.54,2.57)\end{array}$ & $\begin{array}{c}89.06 \\
(12.96,2.20)\end{array}$ & $\begin{array}{c}89.37 \\
(10.96,2.18)\end{array}$ & $\begin{array}{c}91.06 \\
(12.08,2.14)\end{array}$ & 4.12 & .003 & .10 \\
\hline VP & $\begin{array}{c}93.76 \\
(13.00,2.42)\end{array}$ & $\begin{array}{c}82.72 \\
(11.68,2.43)\end{array}$ & $\begin{array}{c}87.09 \\
(14.75,2.07)\end{array}$ & $\begin{array}{c}89.97 \\
(9.80,2.06)\end{array}$ & $\begin{array}{c}91.08 \\
(12.12,2.02)\end{array}$ & 2.44 & .050 & .06 \\
\hline CIT & $\begin{array}{c}90.32 \\
(12.98,2.19)\end{array}$ & $\begin{array}{c}81.20 \\
(10.56,2.19)\end{array}$ & $\begin{array}{c}92.37 \\
(10.72,1.87)\end{array}$ & $\begin{array}{c}91.77 \\
(9.80,1.86)\end{array}$ & $\begin{array}{c}93.19 \\
(10.92,1.83)\end{array}$ & 5.26 & .001 & .12 \\
\hline
\end{tabular}

Nota. La variable sexo fue tenida en cuenta como covariable

Tabla 3. Análisis de Discrepancias Intragrupo-Comparación entre Pares de Índices

\begin{tabular}{|c|c|c|c|c|c|c|}
\hline \multirow[b]{3}{*}{ Índices } & \multicolumn{6}{|c|}{ Diagnóstico } \\
\hline & DAL & TEL & SA & TDAH-I & TDAH-C & Total \\
\hline & $\begin{array}{c}\mathrm{M} \\
\text { (DT, ET) }\end{array}$ & $\begin{array}{c}\mathrm{M} \\
\text { (DT, ET) }\end{array}$ & $\begin{array}{c}\mathrm{M} \\
\text { (DT, ET) }\end{array}$ & $\begin{array}{c}\mathrm{M} \\
(\mathrm{DT}, \mathrm{ET})\end{array}$ & $\begin{array}{c}\mathrm{M} \\
(\mathrm{DT}, \mathrm{ET})\end{array}$ & $\begin{array}{c}\mathrm{M} \\
(\mathrm{DT}, \mathrm{ET})\end{array}$ \\
\hline CV-RP & $\begin{array}{c}-1.24 \\
(15.13,3.03)\end{array}$ & $\begin{array}{c}-7.48 \\
(16.17,3.23)\end{array}$ & $\begin{array}{c}5.43 \\
(12.94,2.19)\end{array}$ & $\begin{array}{c}3.40 \\
(18.27,3.08)\end{array}$ & $\begin{array}{c}3.58 \\
(15.88,2.65)\end{array}$ & $\begin{array}{c}1.41 \\
(16.20,1.30)\end{array}$ \\
\hline CV-MT & $\begin{array}{c}7.88 \\
(13.70,2.74)\end{array}$ & $\begin{array}{c}8.52 \\
(13.58,2.72)\end{array}$ & $\begin{array}{c}13.51 \\
(17.77,3.00)^{* * *}\end{array}$ & $\begin{array}{c}10.91 \\
(17.08,2.89)^{*}\end{array}$ & $\begin{array}{c}10.39 \\
(16.14,2.69)^{*}\end{array}$ & $\begin{array}{c}10.51 \\
(15.92,1.28)\end{array}$ \\
\hline CV-VP & $\begin{array}{c}1.44 \\
(16.3,3.26)\end{array}$ & $\begin{array}{c}3.88 \\
(15.27,3.05)\end{array}$ & $\begin{array}{c}15.49 \\
(18.66,3.15)^{* * *}\end{array}$ & $\begin{array}{c}10.31 \\
(20.23,3.42)\end{array}$ & $\begin{array}{c}10.36 \\
(18.11,3.02)\end{array}$ & $\begin{array}{c}9.03 \\
(18.47,1.48)\end{array}$ \\
\hline RP-MT & $\begin{array}{c}9.12 \\
(18.68,3.74)\end{array}$ & $\begin{array}{c}16.00 \\
(17.83,3.57) * * *\end{array}$ & $\begin{array}{c}8.09 \\
(17.31,2.93)\end{array}$ & $\begin{array}{c}7.51 \\
(15.35,2.60)\end{array}$ & $\begin{array}{c}6.81 \\
(14.62,2.44)\end{array}$ & $\begin{array}{c}9.10 \\
(16.69,1.34)\end{array}$ \\
\hline MT-VP & $\begin{array}{c}-6.44 \\
(15.95,3.19)\end{array}$ & $\begin{array}{c}-4.64 \\
(13.68,2.74)\end{array}$ & $\begin{array}{c}1.97 \\
(18.78,3.18)\end{array}$ & $\begin{array}{c}-.60 \\
(14.77,2.50)\end{array}$ & $\begin{array}{c}-.03 \\
(16.13,2.69)\end{array}$ & $\begin{array}{c}-1.47 \\
(16.16,1.29)\end{array}$ \\
\hline RP-VP & $\begin{array}{c}2.68 \\
(20.65,4.13)\end{array}$ & $\begin{array}{c}11.36 \\
(14.48,2.90)^{*}\end{array}$ & $\begin{array}{c}10.06 \\
(15.69,2.65)\end{array}$ & $\begin{array}{c}6.91 \\
(11.67,1.97)\end{array}$ & $\begin{array}{c}6.78 \\
(15.78,2.63)\end{array}$ & $\begin{array}{c}7.62 \\
(15.70,1.26)\end{array}$ \\
\hline
\end{tabular}

Nota. Puntuaciones Medias de las Discrepancias entre Pares de Índices y sus Niveles de Significatividad en relación a los Valores Críticos establecidos en Wechsler (2003b)

$* p<.15, * * * p<.05$

significativamente bajas respecto a la población normativa; no obstante, el grupo DAL mantiene intacto el índice VP, al igual que ocurre con el grupo TEL en CV.

En la Tabla 2 se muestran los resultados del MANOVA realizado sobre las puntuaciones de los índices del WISC-IV. La prueba de Levene demostró que las varianzas de los grupos en los diferentes índices son similares arrojando los siguientes valores de significación: $\mathrm{CV}=.135$, $\mathrm{RP}=.240, \mathrm{MT}=.380, \mathrm{VP}=.071$ y $\mathrm{CIT}=.640$. Por otra parte, el MANOVA revela diferencias significativas en los índices según el diagnóstico $\left(F_{(146,4)}=2.22\right.$, $p=.002, \eta^{2}=.069$ ). Como se puede observar en la Tabla 2, estas diferencias se concretan en todos los índices excepto en RP. La prueba post-hoc de Bonferroni revela que las diferencias en $\mathrm{CV}$ se dan entre TEL y SA $(p=.000)$, TEL y TDAH-I
( $p=.001)$ y TEL y TDAH-C $(p=.000)$; en MT se ven diferencias entre TEL y SA $(p=.011)$, TEL y TDAH-I $(p=.020)$ y TEL y TDAH-C $(p=.003)$; en VP se aprecian diferencias entre TEL y DAL $(p=.036)$; $y$ en CIT existen diferencias entre TEL y DAL $(p=.050)$, TEL y SA $(p=.001)$, TEL y TDAH-I $(p=.006)$, TEL y TDAH-C $(p=.001)$. Estos datos corroboran parcialmente la $\mathrm{H} 2$ ya que, aunque el MANOVA prueba la existencia de diferencias entre trastornos con un tamaño del efecto grande, la prueba Bonferroni muestra que tales diferencias solo permiten distinguir el perfil TEL del resto de trastornos.

\section{Análisis Intragrupo}

Por su parte, en la Tabla 3 aparecen los resultados obtenidos a partir del proceso de comparación entre pares de índices. Así, se 
presenta la media de la diferencia obtenida por cada grupo diagnóstico en cada pareja de índices junto con su desviación típica, el error típico y la significatividad. De esta forma, se observa que en un nivel de confianza del $85 \%$ no existen diferencias significativas entre las parejas de índices en los sujetos DAL. Por otro lado, en los sujetos TEL sí aparece significatividad entre los pares de índices RP-MT y RP-VP a favor de RP en ambos casos. Del mismo modo, los sujetos SA presentan discrepancias significativas entre los pares CV-MT y CV-VP, en todos los casos mostrando una diferencia positiva en favor del primer índice de la comparación. Por su parte, los sujetos con TDAH-I solo manifiestan diferencias significativas entre CV-MT en favor del primero, al igual que ocurre con el grupo TDAH-C. Atendiendo al nivel de confianza del $95 \%$, se observa que la significatividad varía considerablemente, reduciéndose a los pares RPMT en el caso de los sujetos TEL, así como CVMT y CV-VP en los sujetos SA.

\section{Discusión}

El principal objetivo de este estudio era indagar las posibilidades del WISC-IV como herramienta diagnóstica y de diseño de intervenciones adaptadas a niños con distintos tipos de TND a partir del análisis de la variabilidad intergrupo e intragrupo de sus perfiles. Para ello, en primer lugar, se han evaluado los perfiles cognitivos concretos de los TND seleccionados, estableciendo diferencias intergrupo que permitieran afinar su diagnóstico. A este respecto, los resultados obtenidos con la variedad de trastornos que se han incluido en el estudio aportan claves que complementan el conocimiento que ya existe sobre esta temática. Así, se ha comprobado que existen importantes diferencias entre el perfil de niños TEL y el resto de los trastornos estudiados (principalmente SA, TDAH-I y TDAH-C). Igualmente, se han hallado diferencias significativas entre TEL y DAL en VP lo cual complementa las conclusiones de Schuchardt et al. (2013) añadiendo que el estudio de la VP puede ayudar a discernir entre ambos trastornos. Sin embargo, no se han encontrado grandes diferencias entre los demás diagnósticos ya que las puntuaciones en los índices son bastante similares y se mantienen dentro de la norma. Igualmente, cabe señalar que los grupos TDAH-I y TDAH-C de esta muestra han presentado perfiles idénticos con la misma tendencia en todos los índices, con lo cual en este estudio no se confirma la afectación de estos sujetos en ningún índice del WISC-IV (Bustillo \& Servera, 2015; Fenollar-Cortés et al., 2015) ni la presencia de diferencias entre perfiles (CapdevilaBrophy et al., 2006; Fenollar-Cortés et al., 2015). Asimismo, resulta llamativa la falta de evidencia de déficits en el grupo TEL en el índice CV, cuya existencia ha sido sobradamente probada en estudios previos (Spanoudis \& Natsopoulos, 2011; Taylor, Maybery, \& Whitehouse, 2012; van Weerdenburg et al., 2006). En este estudio la media en este índice se encuentra en el límite de la normalidad, aunque muestra diferencias significativas con el resto de los trastornos, a excepción de DAL. Por ello, en línea con la propuesta de Miller \& Gilbert (2008), se recomienda el uso de diferentes escalas para obtener un perfil más completo y contrastado. Concretamente en el caso de niños con TEL, se aconseja el empleo de escalas de lenguaje complementarias.

Por otro lado, este trabajo revela que el análisis de discrepancias entre pares de índices resulta una interesante fuente de datos que permite ahondar en las diferencias intragrupo y, de este modo, conocer no solo los puntos fuertes y débiles asociados a cada trastorno, sino también, qué índices muestran más disparidad, lo cual proporciona una valiosa referencia con vistas a la intervención. Las repercusiones de estos resultados en la práctica clínica parecen evidentes; si bien la literatura científica destacaba una afectación generalizada en los índices MT y VP entre los trastornos que han formado parte del estudio, a partir del análisis de discrepancias se ha comprobado que dicha afectación resulta aún más significativa en comparación al notable rendimiento en $\mathrm{CV}$ y $\mathrm{RP}$ en prácticamente todos los casos. De este modo, se ha observado que las discrepancias más significativas en un intervalo del 95\% de confianza se han obtenido en la población TEL y SA entre sus índices de mejor rendimiento ( $\mathrm{RP}$ en el primer caso y $\mathrm{CV}$ en el segundo) y aquellos en los que presentan más 
dificultades (MT en el primer caso, y MT y VP en el segundo). Esta tendencia se mantiene si se atiende al intervalo de confianza del $85 \%$, observando cómo se amplía el número de discrepancias significativas, aunque siempre en concordancia con los puntos fuertes y débiles de cada trastorno. Igualmente, en línea con las conclusiones de Nedelcu \& Buceta (2012) esta muestra de SA presenta un patrón de CV superior a RP, aunque el análisis de discrepancias no arroja significatividad estadística en esta diferencia.

Por su parte, el único trastorno que no muestra discrepancias significativas al comparar sus pares de índices es DAL, esto unido al hecho de que sus puntuaciones en cada uno de los índices analizados de manera aislada permanecen dentro de la media normativa, permite inferir que de todos los trastornos estudiados DAL es el que presenta un perfil más homogéneo y con menor afectación, lo cual coincide con las conclusiones de Berninger y O’Donell (2005). A continuación, se encuentran ambos subtipos de TDAH, que muestran disparidad entre algunos de sus índices en un intervalo de confianza del $85 \%$; sin embargo, dicha disparidad desaparece al $95 \%$ de confianza y sus puntuaciones se mantienen dentro de la norma en todos los índices evaluados. Finalmente, los perfiles intragrupo más heterogéneos se observan entre las poblaciones TEL y SA, dado que, tanto en la comparación entre pares de índices como en el análisis individual de los mismos, han obtenido puntuaciones más variables. Estos datos en conjunto permiten confirmar la $\mathrm{H} 3$ que sugería que la población DAL tendría un perfil más homogéneo en tanto que SA mostraría la mayor heterogeneidad; si bien no se esperaba encontrar tal heterogeneidad entre la población TEL, estas afirmaciones concuerdan con las arrojadas por Alloway et al. (2009) y Lum et al. (2015) en el caso de TEL, así como González-Gadea et al. (2014) en el caso de SA.

Para concluir, es importante recalcar la necesidad de ser prudentes a la hora de interpretar y utilizar los resultados derivados de las escalas psicométricas ya que, si bien proporcionan una información muy valiosa tanto a nivel intersujeto como intrasujeto, debido a la multiplicidad de comparaciones realizadas se incrementan los niveles de error tipo 1. Además, es preciso tener en cuenta que existen diversos enfoques para comparar los resultados obtenidos con las tasasbase y, de este modo, establecer la estimación de la anormalidad en base a la distribución de las diferencias en la muestra estandarizada (Crawford et al., 2007; Crawford, Garthwaite, \& Slick, 2009; Crawford et al., 2010). Por todo ello, se recomienda utilizar este tipo de datos con cautela y principalmente como herramientas que faciliten el proceso diagnóstico y orienten las intervenciones.

Finalmente, cabe destacar como limitaciones del estudio el tamaño de la muestra que, debido a su carácter clínico, es más reducido que en otro tipo de trabajos. Por otra parte, sería interesante continuar esta línea de investigación complementando la escala utilizada con otras baterías que midan con mayor precisión aspectos cognitivos específicos tales como el lenguaje. De este modo, se podría profundizar sobre las diferencias encontradas. Igualmente, el análisis de dos índices alternativos creados por Labin, Brenlla, \& Taborda (2018) para evaluar las habilidades fluidas verbales y no verbales a partir del WISC-IV podría emplearse para llevar a cabo un estudio más exhaustivo de estos trastornos. Por último, la introducción, aunque todavía con un uso clínico poco extendido, de la nueva versión de las escalas de Wechsler (WISC-V), ofrece nuevas posibilidades para continuar esta línea de trabajo a fin de conocer si los cambios introducidos en esta versión (principalmente la reconceptualización de algunos índices, incluyendo el visoespacial -VS- y razonamiento fluido -RF-) podrían alterar $\mathrm{o}$ complementar el conocimiento que se tiene hasta la fecha sobre los perfiles cognitivos de estas poblaciones. Los citados índices derivan de los subtests que componen RP en el WISC-IV (Weiss, Saklofske, Holdnack, \& Prifitera, 2016) y dado que éste ha sido el índice que presenta más homogeneidad tanto a nivel intergrupo como intragrupo en este estudio, la replicación de los datos presentados a partir del uso de los índices alternativos del WISC-IV así como del WISC-V podría arrojar nuevas evidencias.

\section{Referencias}

Alloway, T. P., Rajendran, G., \& Archibald, L. (2009). Working memory in children with 
developmental disorders. Journal of Learning Disabilities, 42 , 372-382. doi:10.1177/0022219409335214

American Psychiatric Association. (2013). DSM5. Diagnostic and statistical manual of mental disorders (5thEdition). Washington, DC: Author.

Berninger, V. W., \& O’Donell, L. (2005). Research-supported differential diagnosis of specific learning disabilities. En A. Prifitera, D. H. Saklofske, \& L. G. Weiss, WISC-IV Clinical Use and Interpretation: ScientistPractitioner Perspectives (págs. 189-233). Amsterdam: Elsevier Academic Press.

Bustillo, M., \& Servera, M. (2015). Análisis del patrón de rendimiento de una muestra de niños con TDAH en el WISC-IV. Revista Psicológica Clínica con Niños y Adolescentes, 2(2), 121-128. Recuperado de https://www.redalyc.org/pdf/4771/477147186 005.pdf

Capdevila-Brophy, C., Artigas-Pallarés, J., \& Obiols-Llandrich, J. E. (2006). Tempo cognitivo lento: ¿Síntomas del trastorno de déficit de atención/hiperactividad predominantemente desatento o una nueva entidad clínica?. Revista de Neurología, 42(2), 127-134. doi:10.33588/rn.42S02.2005820

Castro-Rebodello, R., Giraldo-Prieto, M., Hincapié-Henao, L., Lopera, F., \& Pineda, D. A. (2004). Trastorno específico del desarrollo del lenguaje: Una aproximación teórica a su diagnóstico, etiología y manifestaciones clínicas. Revista de Neurología, 39(12), 11731181. doi:10.33588/rn.3912.2004337

Conti-Ramsden, G. (2003). Processing and linguistic markers in young children with specific language impairment. Journal of Speech, Language, and Hearing Research, 46(5), 1029-1037. doi:10.1044/1092-4388(2003/082)

Cornoldi, C., Giofre, D., Orsini, A., \& Pezzuti, L. (2014). Differences in the intellectual profile of children with intellectual vs. learning disability. Research in Developmental Disabilities, 35(9), 2224-2230. doi:10.1016/j.ridd.2014.05.013

Corral, S., Arribas, D., Santamaría, P., Sueiro, M. J., \& Pereña, J. (2005). WISC-IV (Escala de Inteligencia de Wechsler para Niños-IV).
Manual de Aplicación y Corrección. Madrid: TEA Ediciones, S.A.

Crawford, J. R., Anderson, V., Rankin, P. M., \& MacDonald, J. (2010). An index-based shortform of the WISC-IV with accompanying analysis of the reliability and abnormality of differences. British Journal of Clinical Psychology, 49, 235-258. doi:10.1348/014466509X455470

Crawford, J. R., Garthwaite, P. H., \& Gault, C. B. (2007). Estimating the percentage of the population with abnormally low scores (or abnormally large score differences) on standardized neuropsychological test batteries: A generic method with applications. Neuropsychology, 21(4), 419-430. doi:10.1037/0894-4105.21.4.419

Crawford, J. R., Garthwaite, P. H., \& Slick, D. J. (2009). On percentile norms in neuropsychology: Proposed reporting standards and methods for quantifying the uncertainty over the percentile ranks of test scores. The Clinical Neuropsychologist, 23(7), 1173-1195. doi:10.1080/13854040902795018

Evers, A., Muñiz, J., Bartram, D., Boben, D., Egeland, J., Fernández-Hermida, J. R., et al. (2012). Testing practices in the 21st century. European Psychologist, 17(4), 300-319. doi:10.1027/1016-9040/a000102

Fenollar-Cortés, J., Navarro-Soria, I., GonzálezGómez, C., \& García-Sevilla, J. (2015). Detección de perfiles cognitivos mediante WISC-IV en niños diagnosticados con TDAH: ¿Existen diferencias entre subtipos? Revista de Psicodidáctica, 20(1), 157-176. doi:10.1387/RevPsicodidact.12531

Flanagan, D. P., \& Kaufman, A. S. (2006). Claves para la evaluación con el WISC-IV. Madrid: TEA Ediciones.

García, T., Rodríguez, C., González-Castro, P., Álvarez, D., Cueli, M., \& González-Pienda, J. A. (2013). Funciones ejecutivas en niños y adolescentes con trastorno por déficit de atención con hiperactividad y dificultades lectoras. International Journal of Psychology and Psychological Therapy, 13(2), 179-194. Recuperado

de https://www.redalyc.org/pdf/560/5602741600 3.pdf 
Giofrè, D., \& Cornoldi, C. (2015). The structure of intelligence in children with specific learning disabilities is different as compared to typically development children. Intelligence, $\quad 52, \quad 36-43$. doi:10.1016/j.intell.2015.07.002

Gomez, R., Vance, A., \& Watson, S. D. (2016). Structure of the Wechsler Intelligence Scale for Children - Four Edition in a group of children with ADHD. Frontiers in Psychology, 7, 1-11. doi:10.3389/fpsyg.2016.00737

González-Gadea, M. L., Tripicchio, P., Rattazzi, A., Baez, S., Marino, J., Roca, M., Manes, F., \& Ibanez, A. (2014). Inter-individual cognitive variability in children with Asperger's Syndrom. Frontiers in Human Neuroscience, $\quad 8, \quad 575-585$. doi:10.3389/fnhum.2014.00575

Labin, A., Brenlla, M. E., \& Taborda, A. (2018). Índices alternativos del WISC-IV para la Evaluación del Razonamiento Fluido. Revista Iberoamericana de Diagnóstico y Evaluación - e Avaliação Psicológica, 48(3), 81-90. doi:10.21865/RIDEP48.3.07

Lum, J. A. G., Ullman, M. T., \& Conti-Ramsden, G. (2015). Verbal declarative memory impairments in specific language impairment are related to working memory deficits. Brain and Language, 142, 76-85. doi:10.1016/j.bandl.2015.01.008

Miller, C. A., \& Gilbert, E. (2008). Comparison of performance on two nonverbal intelligence tests by adolescents with and without language impairment. Journal of Communication Disorders, 41, 358-371. doi:10.1016/j.jcomdis.2008.02.003

Miranda-Casas, A., Soriano-Ferrer, M., \& Baixauli-Fortea, I. (2013). Written composition performance of students with attention-deficit/hyperactivity disorder. Applied Psycholinguistics, 34(3), 443-460. doi:10.1017/S0142716411000828

Muñiz, J., \& Fernández-Hermida, J. R. (2010). La opinión de los Psicólogos españoles sobre el uso de los tests. Papeles del Psicólogo, 31(1), 108-121. Recuperado de https://www.redalyc.org/pdf/778/7781244101 1.pdf
Nedelcu, D. G., \& Buceta Cancela, M. J. (2012). El perfil cognitivo de los niños con trastorno de Asperger y autismo de alto funcionamiento. Revista Iberoamericana de Diagnóstico y Evaluación - e Avaliação Psicológica, 34(2), 103-116. Recuperado de https://www.redalyc.org/pdf/4596/459645438 005.pdf

Oliveras-Rentas, R. E., Kenworthy, L., Roberson III, R. B., Martin A., \& Wallace, G. L. (2012). WISC-IV profile in high-functioning autism spectrum disorders: Impaired processing speed is associated with increased autism communication symptoms and decreased adaptive communication ability. Journal of Autism and Developmental Disorders, 42, 665-664. doi:10.1007/s10803-011-1289-7

Planche, P., \& Lemonnier, E. (2012). Children with high-functioning autism and Asperger syndrome: Can we differentiate their cognitive profiles? Research in Autism Spectrum Disorders, 6, 939-948. doi:10.1016/j.rasd.2011.12.009

Rabin, L. A., Paolillo, E., \& Barr, W. B. (2016). Stability in test-usage practices of clinical neuropsychologists in the United States and Canada over a 10-year period: A follow-up survey of INS and NAN members. Archives of Clinical Neuropsychology, 31, 206-230. doi:10.1093/arclin/acw007

Reichenbach, K., Bastian, L., Rohrbach, S., Gross, M., \& Sarrar, L. (2016). Cognitive functions in preschool children with specific language impairment. International Journal of Pediatric Otorhinolaryngology, 86, 22-26. doi:10.1016/j.ijporl.2016.04.011

Schuchardt, K., Bockmann, A. K., Bornemann, G., \& Maehler, C. (2013). Working memory functioning in children with learning disorders and specific language impairment. Topics in Language Disorders, 33(4), 298-312. doi:10.1097/01.TLD.0000437943.41140.36

Spanoudis, G. P., \& Natsopoulos, D. (2011). Memory functioning and mental verbs acquisition in children with specific language impairment. Research in Developmental Disabilities, $\quad 32, \quad$ 2916-2926. doi:10.1016/j.ridd.2011.05.011

Taylor, L. J., Maybery, M. T., \& Whitehouse, A. J. O. (2012). Do children with specific 
language impairment have a cognitive profile reminiscent of autism? A review of the literature. Journal of Autism and Developmental Disorders, 42, 2067-2083. doi:10.1007/s10803-012-1456-5

Van Weerdenburg, M., Verhoeven, L., \& van Balkom, H. (2006). Towards a typology of specific language impairment. Journal of Child Psychology and Psychiatry, 47(2), 176189. doi:10.1111/j.1469-7610.2005.01454.x

Wass, S. V. (2015). Applying cognitive training to target executive functions during early development. Child Neuropsychology: A Journal on Normal and Abnormal Development in Childhood and Adolescence, 21(2), 150-166. doi:10.1080/09297049.2014.882888

Wechsler, D. (2003a). Wechsler intelligence scale for children (4th ed.). San Antonio, TX: The Psychological Corporation.

Wechsler, D. (2003b). WISC-IV technical and interpretive manual. London: Harcourt Assessment.

Weiss, L. G., Saklofske, D. H., Holdnack, J. A., \& Prifitera, A. (2016). WISC-V assessment and interpretation: Scientist-practitioner perspectives. Amsterdam: Elsevier Academic Press. 